\title{
The Concept of the GNSSControl Network Densification with Precise Leveling for Ground Deformation Monitoring
}

\author{
Radoslaw Baryla ${ }^{a}$, Jacek Paziewski ${ }^{\text {b }}$ \\ ${ }^{a}$ Department of Satellite Geodesy and Navigation, University of Warmia and Mazury in Olsztyn, Heweliusza 5 st., 10-719, Olsztyn, Poland \\ a Institute of Geodesy, University of Warmia and Mazury in Olsztyn, Oczapowskiego 1 st., 10-719, Olsztyn, Poland
}

\begin{abstract}
Many natural hazards as well as human activities may induce land subsidence and other disasters threating human life and property. In these cases there is a necessity for monitoring of ground deformation. Determination of deformation indices requires high precision geodetic surveys like static GNSS positioning as well as precise spirit leveling. Static GNSS measurements allow for precise determination of the actual height of the controlled points over the investigated area. In such a case the processed baselines are of several kilometers. This makes possible to establish the GNSS reference points outside the affected area. On the other hand, there is often a need for densification of the controlled points and determination of the deformation indices with higher spatial resolution. In this case a precise geometric leveling can be applied. The paper presents concept developed at the University of Warmia and Mazury in Olsztyn of the deformation monitoring with application of integrated GNSS measurements and precise leveling. The developed methodology was introduced and tested at the area of the open pit mine „KWB Adamów” in Poland.
\end{abstract}

Keywords: satellite leveling; Global Navigation Satellite System (GNSS); deformation monitoring; precise leveling.

\section{Introduction}

Many natural hazards as well as human activities may induce land subsidence and other disasters threating human life and property. In these cases there is a necessity for monitoring of ground deformation. Determination of deformation indices requires high precision geodetic surveys like static GPS positioning as well as precise spirit/geometric leveling.

Precise spirit levelling has been proven useful for local area ground deformation, structural deformation monitoring, as well as crustal movement [1]. This technology allows for high accuracy and reliable determination of the actual heights of the controlled points. Several researches were conducted in the field of new adjustment and analysis methods for deformation determination on the basis of spirit leveling [2-5]. On the other hand, this technology is limited to determination only one of the deformation indices which are vertical displacements. Additionally, when long distance (tens of kilometers) between reference and controlled points are considered, this technology is regarded as laborious and timeconsuming. It should be noted that accuracy of the heights obtained from the geometric levelling decreasewith the baseline length, thus high accurate results are obtained in relatively small areas.

Static GPS measurements allow for coordinate determination with baseline lengths of tens of kilometers with millimeter level accuracy [6]. In order to obtain such accuracy, several hour-long sessions as well as elaboratedGNSS observation post processing strategy is required. In particular applied observation linear combination, ambiguity resolution method, as well as ionospheric and tropospheric mitigation method must be carefully chosen [7]. The application of the new GNSS signals gives the possibility for shortening of the observational session [8-10]. Although GNSS technology plays now the most important role in deformation monitoring and geodynamic studies [11-12], still there are cases when precise geometric levelling is less time consuming. This is especially at relatively small areas and when high accuracy is required.

In this paper we propose a concept of densification of the GNSS network with precise levelling for detecting of ground deformation indices such as vertical displacements. In specific, two approaches were described. The developed methodologies were introduced and tested at the area of the „KWB Adamów” open pit mine in Poland.

Corresponding author: Jacek Paziewski. E-mail address: jacek.paziewski@uwm.edu.pl

http://dx.doi.org/10.3846/enviro.2014.187

(C) 2014 The Authors. Published by VGTU Press. This is an open-access article distributed under the terms of the Creative Commons Attribution License, which permits unrestricted use, distribution, and reproduction in any medium, provided the original author and source are credited. 


\section{Methodology}

Figure 1 presents alocal network for ground deformation monitoring at the "KWB Adamów"open pit mine. Primarily, the presented network was established to carry out GNSS measurements and this technology has beenused for several years since 2008. The local network for ground deformation monitoring is built of two sets of points: 3 reference points (RRxx) and 27 controlled points (KKxx). The former points are established and stabilized outside the considered area and realize the local reference frame for the deformation monitoring. The latter are the points which position is controlled, and at which displacements are computed. At both RRxx and KKxx sites GPS observations are collected. The application of GPS technology makes possible to establish the reference points far away from the affected area. Static GPS measurements allow for precise determination of the actual height of the controlled points in respect to the reference points over the investigated area.
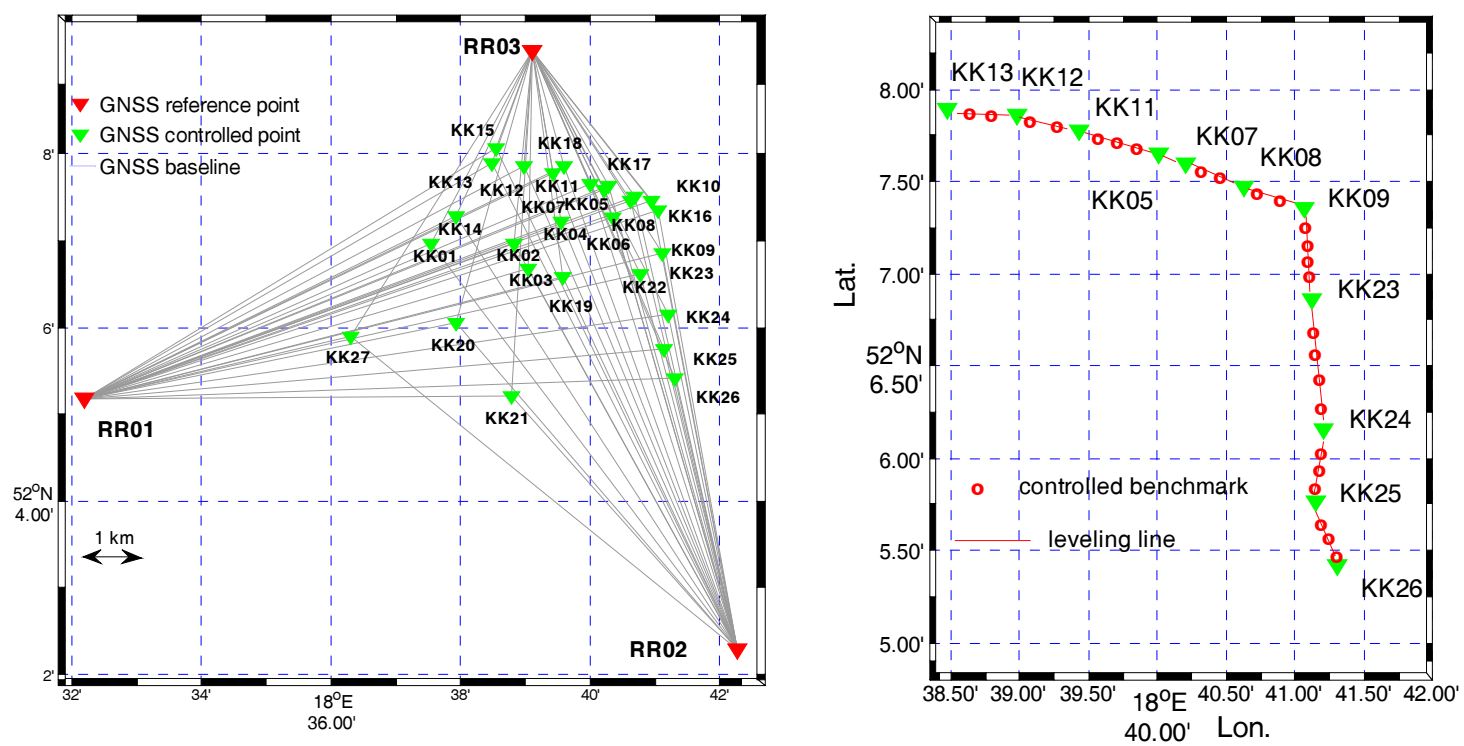

Fig. 1. Local GNSS network for deformation monitoring (left panel) with leveling line densifying GNSS network (right panel)

The research conducted so far raised a need for densification of the control network and determination of the deformation indices with higher spatial resolution in-between KKxx points. For this reason precise geometric spirit leveling was applied, since at short distances $(100-200 \mathrm{~m})$ this technology is less time-consuming.

In order to present the overall concept of the developed methodology, several issues must be described and clarified. The paper concentrates on three aspects of the proposed procedure. Firstly, the principles of the static GPS measurements and spirit levelling measurements with the application of the patented surveying equipment is shown. Secondly, a strategy of the static GPS positioning for satellite levelling in precise local networks is described. Finally, the strategies for integration of the satellite and precise geometric levelling for detection of deformation monitoring are proposed.

\subsection{GNSS and geometric spiritleveling measurements}

At the controlled points GNSS observations are carried out over 2 days, at each day the session lasted 8hours. At the reference points GNSS observations are collected every day during campaign, thus there are six sessions of 8hours. A special attention was paid to the GNSS antennas centering, since this is a very important issuewhen the highest accuracy is to be achieved. The antennas at the reference points were mounted by the means of forced centering at concrete pillars. The height of the antenna over pillar was measured with sub-millimeter precision. The antennas at the controlled points were centered with the application of the patented metal poles. It should be emphasized that at each campaign the same surveying equipment (receiver, antenna and pole) was used. This points to elimination of the biases such as antenna phase center variations and length of the pole when control points heights differences were created.

Precise spirit levelling was carried with the use of the digital level Leica DNA03 with pair of invar rods. The mean length of the line of sight did not exceed $25 \mathrm{~m}$, allowable differences between height differences observed at a station was at the level of $\pm 0.2 \mathrm{~mm}$.

\subsection{GNSS data post processing strategy}

For the GNSS data processing the Bernese 5.0 software was applied. A special strategy for relative static GNSS data processing in precise local networks was developed [13-14]. This strategy was applied for determination of the actual coordinates of the controlled points ( $\mathrm{KKxx}$ ) in each campaign. In the considered network, baseline lengths did not exceed $\sim 15 \mathrm{~km}$. Only independent baselines between RRxx and $\mathrm{KKxx}$ sites were processed taking into account mathematical 
correlations of double-differenced observations. Thus each of the controlled site formed three baselines with reference sites. Since the GNSS baselines length were relatively short, only L1 carrier phase and pseudorange observations were used in the computations. This frequency provides the lowestnoise among available original as well as linear combinations signals. In order to mitigate ionospheric delay, CODE (Center for orbit Determination in Europe) regional model was applied. Another important influence which is tropospheric refraction was modelled by application of the full Saastamoinen model with Niell mapping function. According to our previous research, in small size networks, estimation of the tropospheric zenith delay at sites does not improve positioning results $[13,15]$. The elevation mask was set at the level of $10^{\circ}$. Precise orbits as well as absolute antenna phase center offsets from IGS service were used in the processing [16].

Before determination of the actual coordinates of the controlled points, an analysis of the stability of the reference points is performed at each campaign. It should be noted that this strategy was applied only in local network at subsequent campaigns. The zero-epoch coordinates of the reference points were determined in relation to the IGS sites in the ITRF'05 coordinate frame at the epoch of first campaign. This lead to processing long (over $100 \mathrm{~km}$ ) baselines, thus processing strategy was different, however not presented in this paper.

The application of abovementioned GNSS field measurements and postprocessing strategies allowed for achieving high accuracy of the controlled points coordinates at each campaign. Since coordinates of each controlled point were determined independently twice at each campaign (two sessions), it was possible to compare the solutions obtained for each session. On the basis of the session repeatability at every campaign, it is possible to more reliably estimate the accuracy of the solution. The figure below presents repeatability of the controlled points' coordinates at fifth campaign. It clearly visible that for the most of the coordinate differences were smaller than $2 \mathrm{~mm}$.

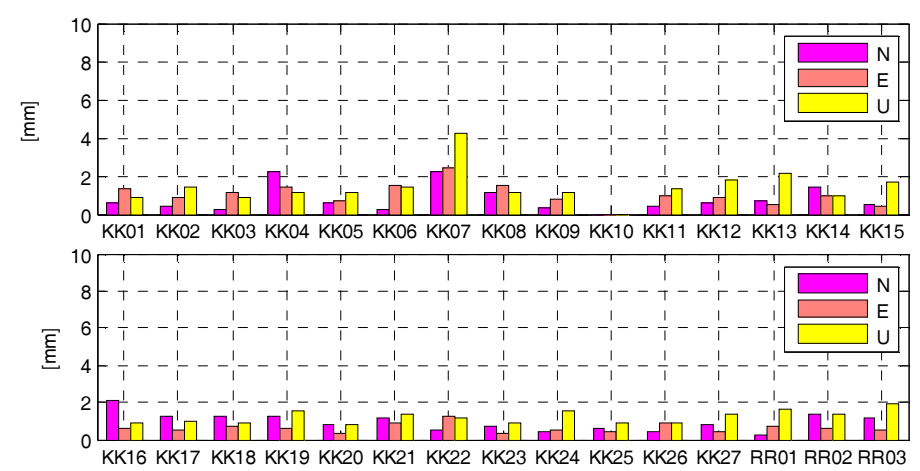

Fig. 2. Repeatability of the controlled points obtained at fifth campaign

\subsection{Processing of the conventional and GNSS data}

Since spirit leveling and GNSS-derived heights are not compatible due to different reference surfaces, special approaches to combine both data must be applied. Height differences derived from spirit leveling are related to the geoid which is equipotential surface, at the same time ellipsoid heights refer to the reference ellipsoid [17]. In order to dense GNSS network with conventional precise leveling two strategies were adopted and developed:

- Densification of GNSS network with conventional levelling,

- Tight integration of the GNSS and spirit leveling for the displacement determination.

\subsubsection{Densification of GNSS network with conventional levelling}

The first strategy relies on the assumption that primarily determined GNSS controlled points KKxx can serve as reference benchmarks for the precise spirit levelling. However, one must remember that position of the former points might change, since these points are at the area of deformation influence. Thus, the actual heights of the KKxx controlled points at the time of campaign are adopted. This way, we obtained two-order network, GNSS network was the first order network, the spirit levelling network was the second order one. In this strategy, the adjustment of GNSS and levelling networks can be performed separately in different coordinate systems.

For the GNSS controlled points, the displacements are computed simply as the coordinate differences in local topocentric coordinate system - North East Up. Vector of the geocentric coordinate differences between the current and zero-epoch campaign is transformed to the local geodetic frame NEU:

$$
\left[\begin{array}{c}
\Delta N_{i} \\
\Delta E_{i} \\
\Delta U_{i}
\end{array}\right]=\left[\begin{array}{ccc}
-\sin \left(B_{i}\right) \cos \left(L_{i}\right) & -\sin \left(B_{i}\right) \sin \left(L_{i}\right) \cos \left(B_{i}\right) \\
-\sin \left(L_{i}\right) & \cos \left(L_{i}\right) & 0 \\
\cos \left(B_{i}\right) \cos \left(L_{i}\right) & \cos \left(B_{i}\right) \sin \left(L_{i}\right) & \sin \left(B_{i}\right)
\end{array}\right] \times\left[\begin{array}{c}
\Delta X_{i} \\
\Delta Y_{i} \\
\Delta Z_{i}
\end{array}\right]
$$


where:

$$
\left[\begin{array}{ccc}
-\sin \left(B_{i}\right) \cos \left(L_{i}\right) & -\sin \left(B_{i}\right) \sin \left(L_{i}\right) & \cos \left(B_{i}\right) \\
-\sin \left(L_{i}\right) & \cos \left(L_{i}\right) & 0 \\
\cos \left(B_{i}\right) \cos \left(L_{i}\right) & \cos \left(B_{i}\right) \sin \left(L_{i}\right) & \sin \left(B_{i}\right)
\end{array}\right] \text { - rotation matrix }
$$

$\Delta N_{i}$
$\Delta E_{i}$ - displacement vector in the local topocentriccoordinate frame, $\left[\begin{array}{c}\Delta X_{i} \\ \Delta Y_{i} \\ \Delta Z_{i}\end{array}\right]$ - geocentric coordinate differences between actual and zero-epoch campaign.

When conventional levelling is applied, adjustment of the height differences is performed in the normal height system. GNSS-derived ellipsoid heights of the KKxx controlled points are converted to the normal heights, which are later held fixed in the adjustment in order to constrain the system. Ellipsoid to normal heights conversion can be performed in several ways, depending on the knowledge of the quasigeoid-ellipsoid separation. In well-known approach, the satellite and geometric levelling integration is performed by transformation of the ellipsoidal heights $\left(h_{i}\right)$ to normal heights $\left(H_{i}\right)$. This is determined by knowledge of the local ellipsoid-quasigeoid separation (height anomalies $\xi_{i}$ ) at each point [18-19]:

$$
H_{i}=h_{i}-\xi_{i}
$$

The height anomalies at each point may be obtained from national or local quasigeoid model by means of interpolation. Another possibility which can be applied for small areas is the application of the one-dimensional surface transformation [20]. The points with known heights in both ellipsoid and normal height systems serve as basis for transformation parameters estimation. Equation 3 presents the simplest transformation with the application of the plane as a surface:

$$
H_{i}=h_{i}+\Delta_{h}-y_{i} \Delta \alpha_{1}+x_{i} \Delta \alpha_{2}
$$

This model contains three parameters: one $\Delta_{h}$ - vertical shift, and two $\Delta \alpha_{1}, \Delta \alpha_{2}$ rotation angles aroundx-axis and $\mathrm{y}$-axis of the local coordinate frame. However, these surface may be used only for small areas, higher order surface transformation should be applied when broaden area is considered.

In both solutions, height accuracy depends on the height conversion process: height anomalies or transformation parameters precision are crucial to obtain accurate result. On the other hand, this is only true if absolute quantities, which are the heights, are considered. In the displacements analysis, the differential values are computed and common bias which is present in both epochs is canceled.

In the second order network, heights of the benchmarks are the parameters in the adjustment and normal height differences between points $i, j$ are the observations in the adjustment algorithm. Height displacements $\Delta_{H_{i}}$ of the controlled benchmarks are computed as a simple normal height difference between actual and zero epoch:

$$
\Delta_{H_{i}}=H^{\prime \prime}{ }_{i}-H^{\prime}{ }_{i}
$$

\subsubsection{Tight integration of the GNSS and spirit leveling for displacements determination}

In the second strategy, a tight integration of the GNSS and conventional levelling is performed. In this approach, there is no need to perform two step adjustment. Thus GNSS reference points RRxx are once held fixed, both KKxx GNSS controlled points, as well as the levelling controlled benchmarks are the determined points. Common adjustment allow for constraining the model. However, the common adjustment of the GNSS and spirit leveling data requires common coordinate system [20]. This lead to a necessity of conversion of the ellipsoidal height differences from GNSS measurements to normal heights.

Here, we propose a new strategy, which can be applied for tight integration of the GNSS and spirit leveling data for the displacement determination without application of common height system. GNSS derived ellipsoid height differences $\left(d h_{e l, i j}\right)$ and spirit leveling-derived normal height differences $\left(d h_{N, i j}\right)$ are the applied original observations. The ellipsoidal height differences are computed simply as the difference between GNSS points' ellipsoidal heights derived from the GNSS network post processing.

$$
d h_{e l, i j}=h_{j}-h_{i}
$$

In the developed approach, we differentiate the original observations (height differences) between epochs (campaigns). In specific, differences of ellipsoid height differences and normal height differences between zero- and subsequent epochs (rate of height differences) serve as new observations. In this relative model, not the heights but the vertical displacements (height changes) of the points are the direct adjusted parameters. When creating original observation differences, common biases for both epochs are cancelled. In particular, there is no need for ellipsoid height difference conversion and common height system introduction since height anomaly in subsequent epochs is the same and is cancelled out. On the other hand, it is important to provide the height differences at the same baselines in each campaign. Below, the functional model of the 
proposed strategy is briefly presented. Eqn (6) shows the observation equation which is created for each ellipsoidal height difference at GNSS baselines:

$$
d h^{\prime \prime}{ }_{e l, i j}-d h_{e l, i j}^{\prime}=h^{\prime \prime}-h^{\prime \prime}{ }_{i} h_{j}^{\prime}+h_{i}^{\prime}
$$

defining vertical displacement as a ellipsoid height difference:

$$
\Delta_{h_{i}}=h^{\prime \prime}{ }_{i}-h_{i}^{\prime}
$$

we obtain the equation where vertical displacements are direct parameters:

$$
d h^{\prime \prime}{ }_{e l, i j}-d h_{e l, i j}^{\prime}=\Delta_{h_{j}}-\Delta_{h i}
$$

where:

$d h^{\prime}{ }_{e l, i j}$ - ellipsoid height difference between $i, j$ points from zero-epoch campaign

$d h^{\prime \prime}{ }_{e l, i j}$ - ellipsoid height difference between $i, j$ points from subsequent campaign

$\Delta_{h_{i}}$ - ellipsoid height change (displacement) between subsequent and zero epoch of theipoint

$\Delta_{h_{j}}$ - ellipsoid height change (displacement) between subsequent and zero epoch of thej point

A similar observation equation is created for each height difference derived from spirit leveling:

$$
d h^{\prime \prime}{ }_{N, i j}-d h_{N, i j}^{\prime}=\Delta_{H_{j}}-\Delta_{H_{i}}
$$

where:

$d h^{\prime}{ }_{N, i j}$ - normal height difference between $i, j$ points from zero-epoch campaign

$d h^{\prime \prime}{ }_{N, i j}$ - normal height difference between $i, j$ points from subsequent campaign

$\Delta_{H_{i}}$ - normal height change (displacement) between subsequent and zero epoch of theipoint

$\Delta_{H_{j}}$ - normal height change (displacement) between subsequent and zero epoch of thej point

Vertical displacements (height changes) of the points are the common desirable parameters in the adjustment. We assume that the displacement of the same point icomputed on the basis of ellipsoid height changes $\left(\Delta_{h_{i}}\right)$ (Eqn 7$)$ and normal height changes $\left(\Delta_{H_{i}}\right)$ (Eqn 4$)$ are equal since for such small height changes plumb line and normal to ellipsoid are close to parallel.

$$
\Delta_{H_{i}} \cong \Delta_{h_{i}}=\Delta_{i}
$$

Combining Eqn $(8,9)$ in a singleobservational model two types of observations, and substituting of ellipsoid height changes $\left(\Delta_{h_{i}}\right)$ and normal height changes $\left(\Delta_{H_{i}}\right)$ by one height change parameter $\left(\Delta_{i}\right)$ (Eqn 10) we obtaina new mathematical model for the combined GNSS and spirit leveling displacement determination (Eqn 11):

$$
\left\{\begin{array}{l}
\Delta_{j}-\Delta_{i}=d h^{\prime \prime}{ }_{e l, i j}-d h_{e l, i j}^{\prime} \\
\Delta_{j}-\Delta_{i}=d h^{\prime \prime}{ }_{N, i j}-d h_{N, i j}^{\prime}
\end{array}\right.
$$

Different stochastic models must be applied for each of the observations. In case of spirit leveling, a priori root mean square error of the height difference over $i, j$ baseline is computed as follows:

$$
m_{i j}=m_{0} \sqrt{D_{i j^{\prime}}+D_{i j "}}
$$

where $m_{0}$ - is the a priori root mean square of unit observation; $D_{i j^{\prime}}$ - is the length of the leveling baseline in zero-epoch campaign, $D_{i j "}$ - is the length of the leveling baseline in subsequent campaign.

When ellipsoidal change of the height difference is regarded, root mean square is computed on the basis of knowledge of the a posteriori root mean square of the GNSS-derived heights of the points at both campaigns:

$$
m_{i j}=\sqrt{m_{h_{i^{\prime \prime}}}^{2}+m_{h_{j^{\prime \prime}}}^{2}+m_{h_{i^{\prime}}}^{2}+m_{h_{j^{\prime}}}^{2}}
$$

The weight of the observation is the inversion of the root mean square of the change of height difference over a baseline (Eqn 14).

$$
p_{i j}=1 / m_{i j}^{2}
$$


In the adjustment, displacements of all points ( $R \mathrm{Rxx}, \mathrm{KKxx}$ and leveling benchmarks) are treated as parameters, however displacements of the reference points (RRxx) are stochastically constrained. Thus, the developed model is adjusted with least squares adjustment with a priori parameter constraining [21]. In this approach, the observational model consists of two groups of observation equations: linearized observation equations with their respective design matrix ( $A$ ), observed minus computed vector $(L)$, and weight matrix $\left(P_{L}\right)$, and pseudo observation equations with their design matrix $(B)$, observed minus computed vector $(W)$, and their weight matrix $\left(P_{W}\right)$. The full weight matrix is constructed with the weight matrix for the actual DD observations $\left(P_{L}\right)$ and the weights of the pseudo observations $\left(P_{w}\right)$. The corrections $\left(d_{X}\right)$ to the a priori values of the parameters are determined resolving well known form of normal equations [20]:

$$
\left(A^{T} P_{L} A+B^{T} P_{W} B\right) d_{X}-\left(A^{T} P_{L} L+B^{T} P_{W} W\right)=0 .
$$

\section{Summary}

The paper presents a concept of the deformation monitoring with application of the integrated GPS and precise leveling measurements developed at the University of Warmia and Mazury in Olsztyn. The methodology was introduced and tested at the area of the open pit mine "KWB Adamów" in Poland. Two different approaches were proposed. The first strategy relies on the assumption that primarily determined GNSS controlled points KKxx can serve as reference benchmarks for the precise spirit levelling. The spirit leveling network is consideredas the second order network. In the second approach, a tight integration of GNSS-derived height differences and levelling-derived height-differences is carried out. In the future research, the two proposed methods will be verified with application of observational data obtained from the area of the "KWB Adamów" open pit mine.

\section{Acknowledgements}

This research was supported by grant agreed by the decision DEC-2011/03/N/ST10/05317 from Polish National Center of Science.

\section{References}

[1] Kowalczyk, K.; Rapinski, J. 2013, Evaluation of levelling data for use in vertical crustal movements model in Poland, Acta Geodynamica et Geomaterialia 10(4): 401-410. http://dx.doi.org/10.13168/AGG.2013.0039

[2] Duchnowski, R. 2011. Robustness of strategy for testing levelling mark stability based on rank tests, Survey Review 43(323): 687-699. http://dx.doi.org/10.1179/003962611X13117748892551

[3] Duchnowski, R. 2013. Hodges-Lehmann estimates in deformation analyses, Journal of Geodesy 87(10-12): 873-884 http://dx.doi.org/10.1007/s00190-013-0651-2

[4] Kaminski, W.; Nowel, K. 2013. Local variance factors in deformation analysis of non-homogenous monitoring networks, Survey Review 45(328): 4450. http://dx.doi.org/10.1179/1752270612Y.0000000019

[5] Hekimoglu, S.; Erdogan, B.; Butterworth, S. 2010. Increasing the efficacy of the conventional deformation analysis methods: alternative strategy, Journal of Surveying Engineering 136: 53-62. http://dx.doi.org/10.1061/(ASCE)SU.1943-5428.0000018

[6] Dach, R.; Hugentobler, U.; Fridez, P.; Meindl, M. 2007. Bernese GPS Software Version 5.0, Astronomical Institute, University of Bern, Jan 2007.

[7] Nyberg, S.; Kallio, U.; Koivula, H. 2013. GPS monitoring of bedrock stability at Olkiluoto nuclear waste disposal site in Finland from 1996 to 2012, Journal of Geodetic Science 3(2): 121-126. http://dx.doi.org/10.2478/jogs-2013-0017

[8] Paziewski, J.; Wielgosz, P.; Krukowska, M. 2013. Application of SBAS pseudorange and carrier phase signals to precise instantaneous singlefrequency positioning, Acta geodynamica et geomaterialia 10(4): 421-430. http://dx.doi.org/10.13168/AGG.2013.0041

[9] Paziewski, J.; Wielgosz, P.2013. Assessment of GPS + Galileo and multi-frequency Galileo single-epoch precise positioning with network corrections, GPS Solutions, online first. http://dx.doi.org/10.1007/s10291-013-0355-3

[10]Chu, F. Y.; Yang, M. 2013. GPS/Galileo long baseline computation: method and performance analyses, GPS Solutions. http://dx.doi.org/10.1007/s10291-013-0327-7

[11]Cai, J.; Wang, J; Wu, J.; Hu, C.; Grafarend, E.; Chen, J. 2008. Horizontal Deformation Rate Analysis Based On Multiepoch GPS Measurements In Shanghai, Journal of Surveying Engineering 134(4): 132-137. http://dx.doi.org/10.1061/(ASCE)0733-9453(2008)134:4(132)

[12] Kontny, B.; Bogusz, J. 2012. Models of vertical movements of the earth crust surface in the area of Poland derived from leveling and GNSS data, Acta Geodynamica et Geomaterialia 9(3): 331-337.

[13]Wielgosz, P.; Paziewski, J.; Baryla, R. 2011. On Constraining Zenith Tropospheric Delays in Processing Of Local GPS Networks With Bernese Software, Survey Review 43(323): 472-483. http://dx.doi.org/10.1179/003962611X13117748891877

[14] Stepniak, K.; Baryła, R.; Wielgosz, P.; Kurpiński, G. 2013. Optimal data processing strategy in precise GPS leveling networks, Acta Geodynamica et Geomaterialia 10(4). http://dx.doi.org/10.13168/AGG.2013.0044

[15]Wielgosz, P.; Paziewski, J.; Krankowski, A.; Kroszczynski, K.; Figurski, M. 2011. Results of the application of tropospheric correctionsfrom different troposphere models for precise GPS rapid staticpositioning, Acta Geophysica 60(4): 1236-1257. http://dx.doi.org/10.2478/s1 1600-011-0078-1

[16]Dow, J. M.; Neilan, R. E.; Rizos, C. 2009. The International GNSS Service in a changing landscape of Global Navigation Satellite Systems, Journal of Geodesy 83: 191-198. http://dx.doi.org/10.1007/s00190-008-0300-3

[17]Hirt, C.; Schmitz, M.; Feldmann-Westendorff, U.; Wubbena, G.; Jahn, C. H.; Seeber, G. 2011. Mutual validation of GNSS height measurementsand high-precision geometric-astronomical leveling, GPS Solutions 15: 149-159. http://dx.doi.org/10.1007/s10291-010-0179-3

[18]Torge, W. 1980. Geodesy. Walter de Gruyter, Berlin New York, 134-137.

[19]Erol, B.; Erol, S.; Celik, R. N. 2008. Height transformation using regional geoids and GPS/levelling in Turkey, Survey Review 40(307): 2-18. http://dx.doi.org/10.1179/003962608X253394

[20]Xu, G. 2007. GPS: Theory, Algorithms and Applications 2nd ed. Berlin Heidelberg Springer-Verlag).

[21]Hofmann - Wellenhof, B.; Lichtenegger, H.; Wasle, E. 2008. GNSS-Global Navigation Satellite Systems GPS, GLONASS, Galileo and more. Springer, Wien- New York. 516 p. 